\title{
Luminescence effects on subcell current-voltage analysis in InGaP/GaAs tandem solar cells
}

\author{
Takeshi Tayagaki,* Ryuji Oshima, Yasushi Shoji, and Takeyoshi Sugaya
}

National Institute of Advanced Industrial Science and Technology, Tsukuba, Ibaraki, Japan

\begin{abstract}
We investigate the role of luminescence effects on the analysis of solar cell properties. InGaP/GaAs tandem solar cells fabricated using hydride vapor phase epitaxy have a luminescent coupling (LC) efficiency of $0.6 \%$ from the top to the bottom subcell. We investigate the impact of LC on subcell current-voltage curve analysis using electroluminescence (EL) measurements. EL efficiency measurements were performed using a reference InGaP single-junction device. It was found that the luminescence extraction from the top subcell, and therefore its luminescence collection efficiency, is lower than that from the bottom subcell. This is due to LC from the top subcell to the bottom subcell. By considering the luminescence extractions of each subcell, more reasonable subcell voltages than those found by conventional methods can be obtained. (C) The Authors. Published by SPIE under a Creative Commons Attribution 4.0 Unported License. Distribution or reproduction of this work in whole or in part requires full attribution of the original publication, including its DOI. [DOI: 10.1117/1.JPE.10.025504]
\end{abstract}

Keywords: current-voltage curve; electroluminescence; luminescent coupling; tandem solar cell.

Paper 20031 received Mar. 23, 2020; accepted for publication May 28, 2020; published online Jun. 5, 2020.

\section{Introduction}

Luminescence properties are very important to consider in the fabrication and characterization of high-efficiency solar cells. ${ }^{1,2}$ Photon recycling has greatly improved the conversion efficiency of thin GaAs solar cells. ${ }^{3,4}$ Multijunction solar cells contain very high-quality materials that exhibit extremely high conversion efficiency and strong luminescent coupling (LC) between the junctions. The presence of LC has a significant impact on the behavior of multijunction solar cells, affecting the optimal design of these devices and thus the characterization of the solar cell.

LC between subcells has been widely studied in stacked series-connected multijunction devices. ${ }^{5-8}$ LC is regarded as a particular case of photon recycling in which luminescence from radiative recombination in a higher-bandgap subcell is reabsorbed by the lower-bandgap subcells, increasing the photocurrent in them. Therefore, LC can overcome the limitations on the current in a series-connected multijunction solar cell caused by lower-bandgap subcells and can increase the current in the entire device. This makes it possible to compensate for current mismatch under spectral mismatch conditions. ${ }^{9}$

LC efficiency has been shown to depend on the properties of the junction interface, such as the device structure ${ }^{10}$ and the air gaps. ${ }^{11,12}$ LC efficiency has been shown to affect the exact characterization of each subcell, such as external quantum efficiency (EQE) measurements. ${ }^{6}$ The LC efficiency of multijunction solar cells has been investigated using various methods, ${ }^{7,13}$ including transient voltage measurements ${ }^{14,15}$ and spectral photovoltage measurements. ${ }^{16}$

Hydride vapor phase epitaxy (HVPE) has received much attention as a low-cost alternative for the fabrication of III-V solar cells. HVPE utilizes cost-effective group-III metals such as gallium and indium and offers high growth rates of several hundred $\mu \mathrm{m} / \mathrm{h}$, which reduces manufacturing cost. InGaP and GaAs single-junction solar cells ${ }^{17,18}$ and an InGaP/GaAs tandem solar cell $^{19}$ have been demonstrated in previous work. To improve the performance of multijunction solar cells, it is necessary to know the basic characteristics of individual subcells, such as the short-circuit current density $\left(J_{\mathrm{sc}}\right)$ and open-circuit voltage $\left(V_{\mathrm{oc}}\right)$. Even though the current-voltage

*Address all correspondence to Takeshi Tayagaki, E-mail: tayagaki-t@aist.go.jp 
curves of individual subcells are not measured directly in series-connected multijunction devices, a combination of electroluminescence (EL) and EQE measurements can be used to describe the subcell current-voltage characteristics.

EL measurements have been used to obtain the subcell $V_{\text {oc }}$ with EQE. ${ }^{20-22}$ The basic theoretical ingredient is Rau's reciprocity relation, the spectral reciprocity relation between solar cells and light-emitting diodes. ${ }^{20}$ Using this, we can estimate $V_{\text {oc }}$ for individual subcells. However, in EL measurements of multijunction devices with efficient LC, applying a forwardbias voltage to the device results in luminescence that generates additional photocurrent in the adjacent lower subcell; this prevents precise characterization of the subcell voltages. ${ }^{23}$ Thus, investigating subcell $V_{\mathrm{oc}}$ is complicated by the LC effect and requires multiple measurements and model analysis. ${ }^{10,23}$

In this study, we investigate the LC efficiency of the top-to-bottom subcells of $\mathrm{InGaP} / \mathrm{GaAs}$ tandem solar cells fabricated using HVPE. In addition, EL efficiency measurements are performed using a reference InGaP single-junction device. Based on these measurements, the luminescence collection efficiency of the subcells is evaluated, and the effect of luminescence extractions for each subcell is discussed. Finally, the subcell voltage obtained by our model is compared with that estimated by conventional methods.

\section{Experimental}

For this study, InGaP/GaAs tandem solar cells and InGaP single-junction devices were fabricated using HVPE. The fabrication process followed the method described in previous papers. ${ }^{17-19,24}$ All samples were grown on a 2-in. diameter GaAs (001) substrate that was miscut 4 deg toward the (111)B direction in the custom hot-wall reactor (Taiyo Nippon Sanso, H260) at atmospheric pressure. For all of the solar cells, the growth rates for GaAs and InGaP were 12 and $24 \mu \mathrm{m} / \mathrm{h}$, respectively. For the InGaP/GaAs solar cell, the thicknesses of the $\mathrm{n}$-InGaP and p-InGaP absorption layers were 200 and $1000 \mathrm{~nm}$, respectively. The $\mathrm{InGaP}$ and GaAs p-n junctions were connected in series through a tunnel junction composed of $20 \mathrm{~nm}$ thick $\mathrm{p}^{+}$-GaAs and $\mathrm{n}^{+}$-GaAs layers. For the $\mathrm{InGaP}$ solar cell, the $\mathrm{p}-\mathrm{InGaP}$ absorption layer thickness is $800 \mathrm{~nm}$. After HVPE growth of the device structures, $\mathrm{AuGeNi} / \mathrm{Au}$ and Ti/Au electrodes were formed as n-type and p-type ohmic contacts using electron-beam evaporation. Mesa isolation was performed using a standard photolithography system. $\mathrm{An} \mathrm{SiO}_{2}(110 \mathrm{~nm}) / \mathrm{TiO}_{2}(50 \mathrm{~nm})$ antireflection coating (ARC) was deposited onto the cell by radio-frequency magnetron sputtering. The device area was $0.1024 \mathrm{~cm}^{2}$. Note that for the reference InGaP solar cell, the front layer conditions such as thicknesses of ARC, $\mathrm{n}-\mathrm{InGaP}$ window layer, and $\mathrm{n}-\mathrm{InGaP}$ emitter set the same parameters as the $\mathrm{InGaP} / \mathrm{GaAs}$ tandem solar cell for comparison. This is because these parameters may affect the extraction efficiency of luminescence that originates from radiative electron-hole recombination at the InGaP p-n junction.

Current-voltage characteristics were measured using a Xe/halogen two-light-source solar simulator. The EQE was measured with chopped, monochromatic light having a constant photon flux of $1 \times 10^{14} \mathrm{~cm}^{-2}$. The EQE curves of tandem solar cells were performed to minimize the artifacts by adjusting the bias light and voltage according to mature procedures. ${ }^{25}$ In the EL intensity measurements, current was injected by applying a constant voltage to the devices. Luminescence was collected using a fiber optic probe and detected by a charge-coupled device.

\section{Results and Discussion}

\subsection{Fundamental Solar Cell Characteristics}

Figure 1(a) shows the current-voltage curves of the InGaP/GaAs tandem solar cell and the reference InGaP solar cell. Current-voltage characteristics of the solar cells were measured using a $\mathrm{Xe} /$ halogen dual-light-source solar simulator. The InGaP/GaAs tandem solar cell used in this work had a $J_{\mathrm{sc}}$ of $11.6 \mathrm{~mA} / \mathrm{cm}^{2}, V_{\mathrm{oc}}$ of $2.30 \mathrm{~V}$, fill factor of 0.81 , and efficiency of $21.7 \%$ at 1 sun. The reference InGaP solar cell had a slightly reduced $J_{\mathrm{sc}}$ compared with the tandem device. 

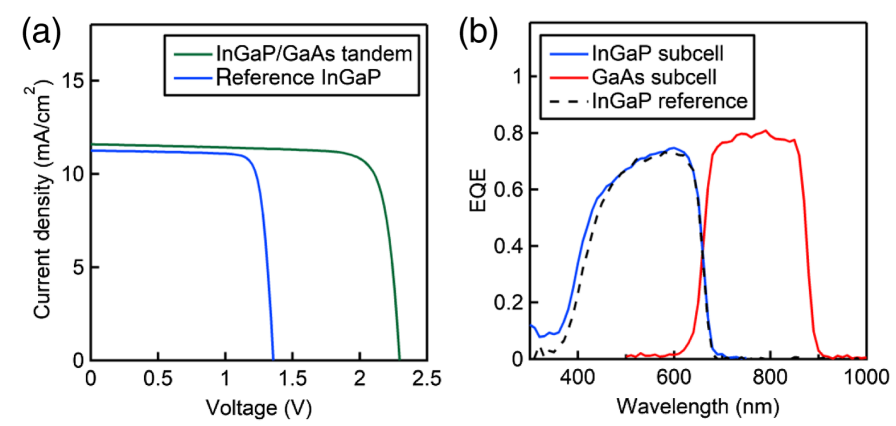

Fig. 1 (a) Current-voltage curves of InGaP/GaAs tandem solar cell and reference InGaP solar cell. (b) EQE curves of the InGaP and GaAs subcells of the InGaP/GaAs tandem solar cell and the reference InGaP solar cell.

Figure 1(b) shows the EQE curves for the InGaP and GaAs subcells of the InGaP/GaAs tandem solar cell and the reference InGaP solar cell. The subcell $J_{\mathrm{sc}}$, as implied by the EQE spectra, was 11.2 and $11.5 \mathrm{~mA} / \mathrm{cm}^{2}$, in the $\mathrm{InGaP}$ top and $\mathrm{GaAs}$ bottom subcells, respectively; this meets the current matching requirements of tandem solar cells. The reference InGaP solar cell had a slightly reduced EQE value at shorter wavelengths (below $500 \mathrm{~nm}$ ) compared with the InGaP subcell. This is probably why the two curves differ in Fig. 1(a).

\subsection{Analysis of Luminescent Coupling Efficiency}

First, we analyze the LC efficiency from the top to the bottom subcell. To estimate the LC, photocurrent was generated only in the top InGaP subcell using monochromatic 405-nm laser illumination. The current in the top subcell was evaluated using the reference InGaP singlejunction device under the same conditions. Figure 2(a) shows the current-voltage curves of the reference InGaP solar cell measured at different 405-nm laser intensities. Thus, the reference
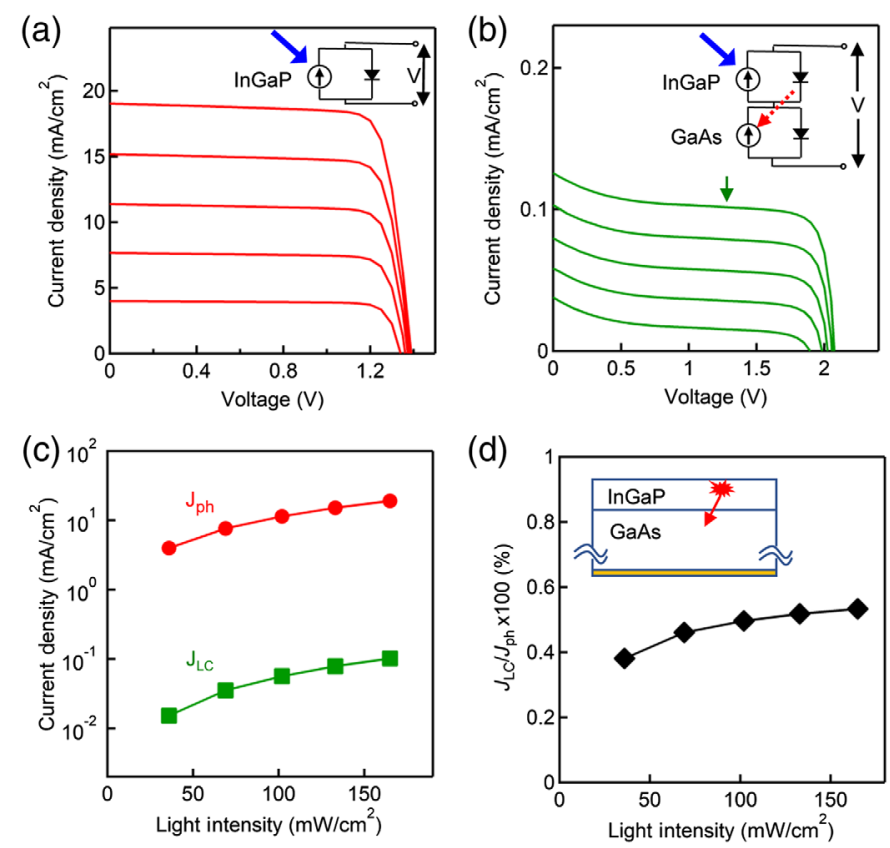

Fig. 2 (a) Current-voltage curves of reference InGaP solar cell and (b) InGaP/GaAs tandem solar cell measured at different 405-nm laser intensities. Inset: Schematic diagram of the equivalent circuit. (c) Photocurrent generated in reference InGaP solar cell and current generated through LC in the InGaP/GaAs tandem solar cell. (d) LC efficiency calculated from (c) as a function of light intensity. Inset: Schematic of LC from InGaP top subcell to GaAs bottom subcell. 
InGaP solar cell generated a photovoltage of $\sim 1.3 \mathrm{~V}$ at a photocurrent of 5 to $20 \mathrm{~mA} / \mathrm{cm}^{2}$. InGaP/GaAs tandem devices consist of a fully absorbing InGaP subcell stacked on a GaAs subcell, with the bottom GaAs subcell generating current from the top InGaP subcell via LC only. The LC current generated by the bottom GaAs subcell limits the current in a series-connected $\mathrm{InGaP} / \mathrm{GaAs}$ tandem device because it is smaller than the current generated directly by laser illumination of the top InGaP subcell.

Figure 2(b) shows the current-voltage curves of an InGaP/GaAs tandem solar cell measured at different 405-nm laser intensities. The current in the figure is the photocurrent into the GaAs subcell of the InGaP/GaAs tandem solar cell generated via LC. The obtained $V_{\text {oc }}$ of $\sim 2 \mathrm{~V}$ indicates that the GaAs subcell shows $V_{\text {oc }}$ of $\sim 0.7 \mathrm{~V}$ due to photocurrent generation via LC because $V_{\text {oc }}$ of $1.3 \mathrm{~V}$ is obtained in the reference InGaP solar cell under the same 405-nm laser illumination and the $V_{\text {oc }}$ of the tandem device is determined by the sum of the subcell $V_{\text {oc }}$. Here, we assume that the photocurrent measured at $1.3 \mathrm{~V}$ is the short-circuit current of the GaAs subcell because the photovoltage of $1.3 \mathrm{~V}$ is generated in the InGaP subcell and the photocurrent of the tandem device is limited by the GaAs subcell. The increase in current with decreasing voltage below $1.3 \mathrm{~V}$ is probably related to an increase in dark current in the GaAs subcell toward GaAs diode breakdown.

Figure 2(c) shows the current generated by the GaAs subcell of the InGaP/GaAs tandem solar cell and the reference InGaP solar cell. In the reference single-junction InGaP device, the current increases in proportion to the illumination. Figure 2(d) shows $J_{\mathrm{LC}} / J_{\mathrm{ph}}$, the ratio between the current densities in the InGaP/GaAs tandem device and the reference InGaP device, obtained from Fig. 2(c). The current ratio $J_{\mathrm{LC}} / J_{\mathrm{ph}}$ reflects the LC efficiency of the $\mathrm{InGaP} / \mathrm{GaAs}$ tandem solar cell from the top subcell to the bottom subcell. LC efficiency increases slightly with the light intensity of the illumination. This increase has been interpreted as meaning that the top subcell becomes more radiative with increasing illumination intensity. This increase in radiative efficiency is explained by the fact that the Shockley-Read-Hall lifetimes of electron and hole increase with illumination intensity. ${ }^{5}$ The measured current ratio was of $0.4 \%$ to $0.6 \%$ and remained almost unchanged above $100 \mathrm{~mW} / \mathrm{cm}^{2}$, showing that the LC occurs in the tandem device fabricated using HVPE.

Even though the luminescent effect is a small contributor to the efficiency of the bottom subcell, it would be able to design a device to increase LC efficiency by enhancing the EQE of the GaAs subcell around the peak wavelength of EL of the $\mathrm{InGaP}$ layer around $650 \mathrm{~nm}$. This can be achieved by the thinning InGaP layer, which increases the EQE of the GaAs subcell around $650 \mathrm{~nm}$ because the $\mathrm{EQE}$ of the GaAs subcell is determined by the transmittance of the incident light through the InGaP subcell. In Fig. 1(b), the wavelength light shorter than $650 \mathrm{~nm}$ is absorbed by the front InGaP layer and is not transmitted to the GaAs layer, resulting in a reduced EQE of the GaAs subcell below $650 \mathrm{~nm}$. The modification of the InGaP subcell thickness would probably increase in LC efficiency from the InGaP subcell to the GaAs subcell.

\subsection{Current-Voltage Analysis Using Electroluminescence Measurements}

Next, we perform an analysis of the subcell $V_{\mathrm{oc}}$ and investigate the effect of LC on the analysis. Subcell $V_{\mathrm{oc}}$ was calculated from the EL intensity at a given current according to the procedure reported in previous papers. ${ }^{20,21}$ Figure 3 (a) shows the dark current-voltage curves of the $\mathrm{InGaP} /$ GaAs tandem and reference InGaP solar cells. The markers in Fig. 3(a) indicate the injection currents used for the EL measurements. Figure 3(b) shows the EL spectra of the InGaP/GaAs tandem solar cell measured at different injection currents. The luminescence signals at 660 and $870 \mathrm{~nm}$ correspond to InGaP and GaAs, respectively. Figure 3(c) summarizes the EL intensity of the InGaP and $\mathrm{GaAs}$ subcells of the tandem and reference InGaP solar cells. The luminescence intensity of both subcells increased with the injection current.

The current-voltage curves of the individual subcells are evaluated from EL and EQE measurements using the spectral reciprocity relation between the solar cell and light-emitting $\operatorname{diode}^{21}$

$$
\varphi_{\mathrm{EL}, i}\left(J_{\mathrm{EL}}\right)=\varphi_{\mathrm{EQE}, i} \cdot \varphi_{\mathrm{BB}}\left\{\exp \left[\frac{q V_{i}\left(J_{\mathrm{EL}}\right)}{k T}\right]-1\right\},
$$


Tayagaki et al.: Luminescence effects on subcell current-voltage analysis...
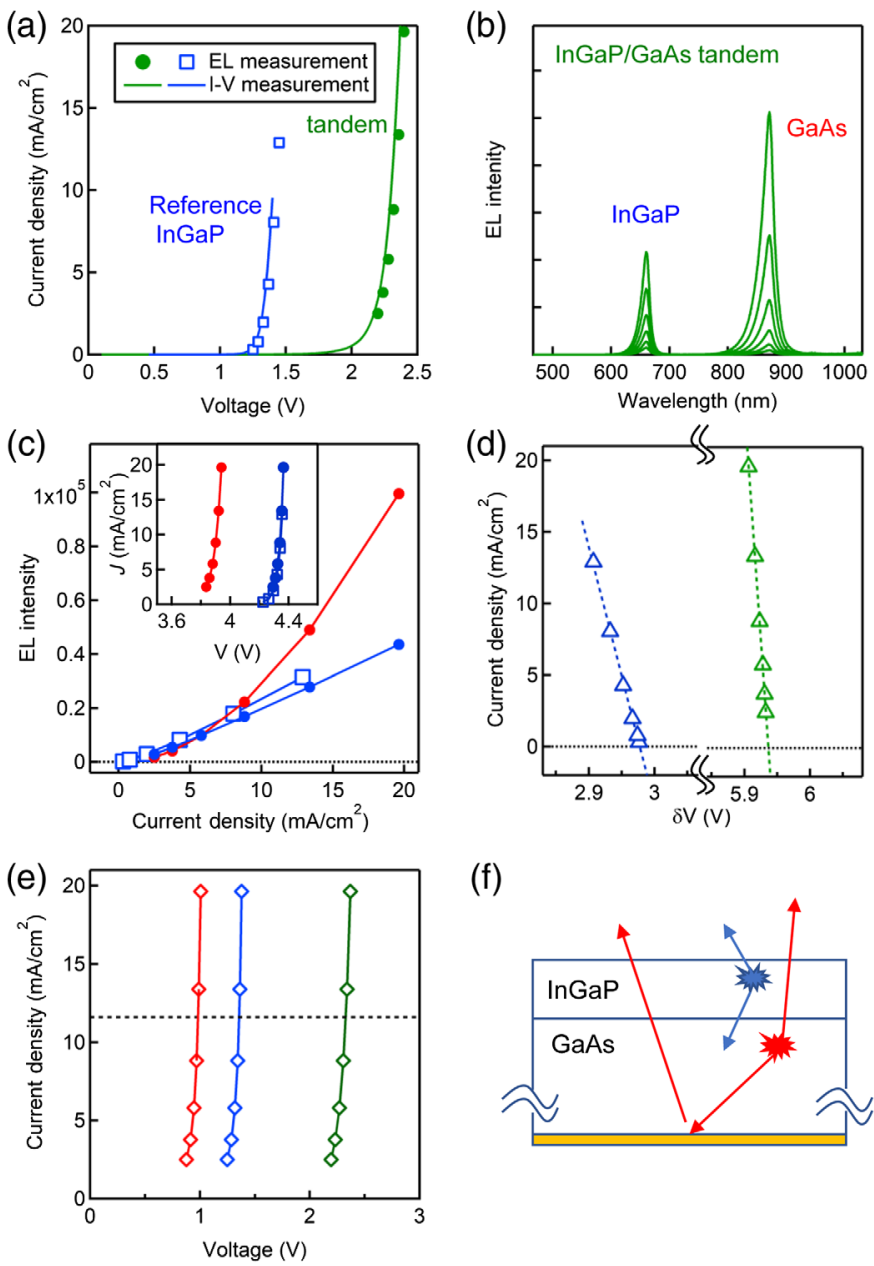

(f)

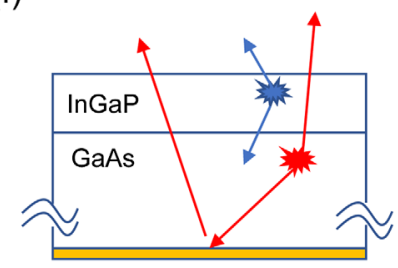

Fig. 3 (a) Dark current-voltage curves of InGaP/GaAs tandem and reference InGaP solar cells. (b) EL spectra of InGaP/GaAs tandem measured at different injection currents. (c) EL intensity of InGaP (blue solid circle) and GaAs (red solid circle) subcells of tandem and reference InGaP solar cells (blue open square). Inset: $V_{i}^{*}$ of the InGaP and GaAs subcells of the tandem and the reference InGaP devices calculated using Eq. (2). (d) Calculated offset voltage of reference InGaP (blue triangle) and tandem solar cells (green triangle) at different injection currents. (e) Currentvoltage curves of $\mathrm{GaAs}$ (red diamond) and InGaP (blue diamond) subcells and the tandem solar cells (green diamond) obtained by EL intensity analysis. (f) Schematic diagram of LC effect of InGaP/GaAs tandem solar cell.

where $\varphi_{\mathrm{EL}, i}\left(J_{\mathrm{EL}}\right)$ is the intensity of the EL peak signal of the subcell $i$, a function of the EL injection current density $J_{\mathrm{EL}} ; \varphi_{\mathrm{EQE}, i}$ and $\varphi_{\mathrm{BB}}$ represent the EQE and black body photon fluxes with respect to the photon energy $E$ of the EL peak of the subcell $i ; q$ is the elementary charge; and $V_{i}$ is the current-density-dependent voltage of the subcell $i$. Using the Boltzmann approximation, Eq. (1) is rearranged as ${ }^{21}$

$$
V_{i}\left(J_{\mathrm{EL}}\right)=\frac{k T}{q} \ln \left[\varphi_{\mathrm{EL}, i}\left(J_{\mathrm{EL}}\right)\right]+\frac{E}{q}-\frac{k T}{q} \ln (E)-2 \frac{k T}{q} \ln \left(\varphi_{\mathrm{EQE}, i}\right)-\delta V_{i}=V_{i}^{*}\left(J_{\mathrm{EL}}\right)-\delta V_{i} .
$$

Equation (2) allows us to determine the subcell voltage $V_{i}$ as a function $V_{i}^{*}$ of $J_{\mathrm{EL}}$, except for the voltage offset $\delta V_{i}$. The first, second, and third terms are taken from the EL measurements, while the fourth term is taken from the EQE measurement. The inset of Fig. 3(c) shows the $V_{i}^{*}$ of the InGaP and GaAs subcells of the tandem and the reference InGaP devices calculated using Eq. (2).

The last term, $\delta V_{i}$, reflects luminescence collection factors, such as geometrical factors that reflect the shape of the optics setup and luminescence extraction efficiency. Previous papers 
assumed that the voltage offsets are the same for all subcells of the device under test. ${ }^{21}$ Here, we estimate the voltage offset using the dark current-voltage curves of the tandem and reference devices. Figure 3(d) shows the voltage offsets of the reference InGaP and tandem solar cells from the results in Fig. 3(a) and the inset of Fig. 3(c) for various injection currents. The voltage offset is slightly dependent on the injection currents. This is probably due to series resistance that influences only current-voltage curve measurements, while it does not affect the curves obtained from the EL intensity. The current-voltage characteristic of each subcell, derived from the EL intensity, represents $J_{\mathrm{sc}}-V_{\mathrm{oc}}$ pairs and therefore is free of influences of series resistance. ${ }^{26}$ By fitting the relation in Fig. 3(d) using a linear function, we obtained the voltage offset $\delta V_{\text {InGaP }}$ of $2978 \mathrm{mV}$ at zero current limit. Similarly, as shown in Fig. 3(d), we analyzed the current-voltage characteristics of the $\mathrm{InGaP} / \mathrm{GaAs}$ tandem solar cell and obtained the voltage offset for the tandem. Here, the voltage of the tandem is the sum of the subcell voltages: $V_{\operatorname{tandem}}\left(J_{\mathrm{EL}}\right)=$ $V_{\mathrm{InGaP} \text { sub }}^{*}\left(J_{\mathrm{EL}}\right)-\delta V_{\mathrm{InGaP} \text { sub }}+V_{\mathrm{GaAs} \mathrm{sub}}^{*}\left(J_{\mathrm{EL}}\right)-\delta V_{\mathrm{GaAs} s u b}$. The voltage offset of the tandem was obtained from the calculated voltage in Fig. 3(c) and the dark current-voltage curve of the tandem device in Fig. 3(a): $\delta V_{\text {InGaaP sub }}+\delta V_{\text {GaAs sub }}$. Fitting the voltage offset using a linear function gave a voltage offset of $5936 \mathrm{mV}$ at zero current limit. By assuming that the voltage offset of the InGaP subcell was the same as the voltage offset of the reference InGaP solar cell, $\delta V_{\text {InGaP sub }}=$ $\delta V_{\text {InGaP }}$, the voltage offset $\delta V_{\text {GaAs sub }}$ was determined to be $2958 \mathrm{mV}$. Note that the voltage offset of the InGaP subcell was larger by $20 \mathrm{mV}$ than the voltage offset of the GaAs subcell. This indicates that the luminescence collection efficiency of the InGaP subcell was low, as will be described later.

Equation (2) gives the current-voltage curves for GaAs and InGaP subcells, shown in Fig. 3(e). Combined with a subcell photocurrent of $11.6 \mathrm{~mA} / \mathrm{cm}^{2}$, a subcell $V_{\mathrm{oc}}$ of $1357 \mathrm{mV}$ was estimated for the top InGaP subcell and $983 \mathrm{mV}$ for the bottom GaAs subcell. Therefore, as shown in Fig. 1(a), the $V_{\mathrm{oc}}$ of the tandem device generated from the internal voltage of the InGaP and GaAs subcells was $2300 \mathrm{mV}$.

Conventional procedures ${ }^{21,22}$ assume that the voltage offsets for all subcells are the same, $\delta V_{\text {InGaP sub }}=\delta V_{\text {GaAs sub }}$. Without considering the voltage offset of each subcell, the individual subcell $V_{\mathrm{oc}} s$ were extracted as 1376 and $962 \mathrm{mV}$ for InGaP and GaAs subcells, respectively. The value of the InGaP subcell was slightly larger than $1350 \mathrm{mV}$, the value of the reference InGaP solar cells reported in a previous paper. ${ }^{18}$ This also means that the luminescence extraction efficiency of the InGaP subcell is lower than that of the GaAs subcell. For multijunction devices with three or more junctions, the effect of the voltage offset difference may be negligible because the voltage offset is averaged between the subcells. However, for double junction devices, these results indicate that the luminescence collection coefficients, such as the luminescence extraction efficiency and geometric factors that reflect the shape of the optical setup, need to be different for each subcell. We carefully checked that the geometric factors caused by the optics setup do not change to obtain exactly the same result for each subcell. Therefore, the discrepancy is probably caused by the luminescence extraction efficiency of the device. A difference in voltage offset of $\sim 20 \mathrm{mV}$ corresponds to a luminescence extraction coefficient of the InGaP subcell that is about half of the GaAs subcell. This cannot be explained by the internal radiative efficiency of the $\mathrm{InGaP}$ subcell alone because it is unlikely to be $<50 \%$ even if we assume the radiative efficiency of GaAs to be $100 \%$. A possible cause is luminescence reabsorption or an LC effect in the GaAs layer behind the InGaP subcell, as shown in Fig. 3(f). Luminescence extraction efficiency is related to the device structures and depends on each device. Thus, our findings indicate that a more detailed analysis can be performed with the help of a reference device and we can evaluate more accurately the efficiency of luminescence extraction.

\section{Conclusion}

We analyzed LC effects in subcell current-voltage analysis of HVPE-based InGaP/GaAs tandem solar cells. Current-voltage characteristics of individual subcells were obtained by EL and EQE measurements. Our findings indicate that luminescence extraction efficiency should be calibrated for each subcell and that EL intensity is affected by LC effects. 
Tayagaki et al.: Luminescence effects on subcell current-voltage analysis...

\section{Acknowledgments}

The authors gratefully acknowledge T. Aihara for experimental support and K. Makita and A. Ubukata for the development and fabrication of the devices. A part of this work was supported by the New Energy and Industrial Technology Development Organization (NEDO) under the Ministry of Economy, Trade and Industry (METI) and KAKENHI (Grant No. 18K04987) from JSPS.

\section{References}

1. D. J. Friedman, J. F. Geisz, and M. A. Steiner, "Effect of luminescent coupling on the optimal design of multijunction solar cells," IEEE J. Photovoltaics 4(3), 986-990 (2014).

2. C. Baur et al., "Effects of optical coupling in III-V multilayer systems," Appl. Phys. Lett. 90(19), 192109 (2007).

3. O. D. Miller, E. Yablonovitch, and S. R. Kurtz, "Strong internal and external luminescence as solar cells approach the Shockley-Queisser limit," IEEE J. Photovoltaics 2(3), 303-311 (2012).

4. M. A. Steiner et al., "Optical enhancement of the open-circuit voltage in high quality GaAs solar cells," J. Appl. Phys. 113(12), 123109 (2013).

5. A. W. Walker et al., "Impact of photon recycling and luminescence coupling on III-V single and dual junction photovoltaic devices," J. Photonics Energy 5(1), 053087 (2015).

6. D. Lan and M. A. Green, "Photoluminescent and electroluminescent couplings in monolithic tandem solar cells," Prog. Photovolt Res. Appl. 24, 1566-1576 (2016).

7. M. A. Steiner et al., "Measuring IV curves and subcell photocurrents in the presence of luminescent coupling," IEEE J. Photovoltaics 3(2), 879-887 (2013).

8. T. Tayagaki et al., "Analysis of luminescence coupling effect in three-terminal tandem solar cells," J. Photonics Energy 8(4), 045503 (2018).

9. A. S. Brown and M. A. Green, "Radiative coupling as a means to reduce spectral mismatch in monolithic tandem solar cell stacks theoretical considerations," in Proc. 29th IEEE Photovolt. Spec. Conf., pp. 868-871 (2002).

10. J. F. Geisz et al., "Generalized optoelectronic model of series-connected multijunction solar cells," IEEE J. Photovoltaics 5(6), 1827-1839 (2015).

11. X. Sheng et al., "Device architectures for enhanced photon recycling in thin-film multijunction solar cells," Adv. Energy Mater. 5, 1400919 (2015).

12. T. Tayagaki et al., "Investigation of the properties of semiconductor wafer bonding in multijunction solar cells via metal-nanoparticle arrays," J. Appl. Phys. 122, 023101 (2017).

13. M. A. Steiner and J. F. Geisz, "Non-linear luminescent coupling in series-connected multijunction solar cells," Appl. Phys. Lett. 100(25), 251106 (2012).

14. H. Nesswetter et al., "Determination of subcell I-V parameters by a pulsed suns-Voc method including optical coupling," Appl. Phys. Lett. 106(2), 023903 (2015).

15. T. Tayagaki et al., "Transient analysis of luminescent coupling effects in multi-junction solar cells," J. Appl. Phys. 124(18), 183103 (2018).

16. D. F. Marrón et al., "Quantitative determination of luminescent coupling in multijunction solar cells from spectral photovoltage measurements," Phys. Rev. Appl. 6, 014001 (2016).

17. R. Oshima et al., "Improvement of heterointerface properties of GaAs solar cells grown with InGap layers by hydride vapor-phase epitaxy," IEEE J. Photovoltaics 9(1), 154-159 (2019).

18. Y. Shoji et al., "Ultrafast growth of InGaP solar cells via hydride vapor phase epitaxy," Appl. Phys. Express 12(5), 052004 (2019).

19. R. Oshima et al., "High doping performance of sulfur and zinc dopants in tunnel diodes using hydride vapor phase epitaxy," IEEE J. Photovoltaics 10(3), 749-753 (2020).

20. U. Rau, "Reciprocity relation between photovoltaic quantum efficiency and electroluminescent emission of solar cells," Phys. Rev. B 76(8), 085303 (2007).

21. T. Kirchartz et al., "Internal voltages in GaInP/GaInAs/Ge multijunction solar cells determined by electroluminescence measurements," Appl. Phys. Lett. 92(12), 123502 (2008).

22. S. Roensch et al., "Subcell I-V characteristic analysis of GaInP/GaInAs/Ge solar cells using electroluminescence measurements," Appl. Phys. Lett. 98(25), 251113 (2011). 
23. J. F. Geisz et al., "Device characterization for design optimization of 4 junction inverted metamorphic concentrator solar cells," AIP Conf. Proc. 1616, 114 (2014).

24. T. Aihara et al., "Analysis of subcell open-circuit voltages of InGaP/GaAs dual-junction solar cells fabricated using hydride vapor phase epitaxy," Jpn. J. Appl. Phys. 59, SGGF02 (2020).

25. M. Meusel et al., "Spectral response measurements of monolithic GaInP/Ga(In)As/Ge triplejunction solar cells: measurement artifacts and their explanation," Prog. Photovoltaics Res. Appl. 11(8), 499-514 (2003).

26. C. Karcher et al., "Temperature-dependent electroluminescence and voltages of multijunction solar cells,” Prog. Photovoltaics Res. Appl. 22, 757-763 (2014).

Biographies of the authors are not available. 